\title{
Revealed preference and satisficing behavior
}

\author{
Robert van Rooij
}

Received: 29 September 2010 / Accepted: 7 October 2010 / Published online: 19 October 2010

(C) The Author(s) 2010. This article is published with open access at Springerlink.com

\begin{abstract}
A much discussed topic in the theory of choice is how a preference order among options can be derived from the assumption that the notion of 'choice' is primitive. Assuming a choice function that selects elements from each finite set of options, Arrow (Economica 26:121-127, 1959) already showed how we can generate a weak ordering by putting constraints on the behavior of such a function such that it reflects utility maximization. Arrow proposed that rational agents can be modeled by such choice functions. Arrow's standard model of rationality has been criticized in economics and gave rise to approaches of bounded rationality. Two standard assumptions of rationality will be given up in this paper. First, the idea that agents are utility optimizers (Simon). Second, the idea that the relation of 'indifference' gives rise to an equivalence relation. To account for the latter, Luce (Econometrica 24:178-191, 1956) introduced semi-orders. Extending some ideas of Van Benthem (Pac Philos Q 63:193-203, 1982), we will show how to derive semi-orders (and so-called interval orders) based on the idea that agents are utility satisficers rather than utility optimizers.
\end{abstract}

Keywords Revealed preference - Satisficing behavior - Comparison relation · Vagueness

\section{Deriving weak orderings}

A much discussed topic in the theory of choice is how a preference order among options can be derived on the assumption that the notion of choice is primitive. Assuming a choice function that selects elements from each finite set of options, Arrow (1959)

R. van Rooij (凶)

Institute for Logic, Language, and Computation, University of Amsterdam, Amsterdam, Netherlands e-mail: R.A.M.vanRooij@uva.nl 
already showed how we can generate a weak ordering by putting constraints on how this function should behave on different sets of options. Let us define a choice structure to be a triple $\langle A, O, C\rangle$, where $A$ is a non-empty set of options, the set $O$ consists of all finite subsets of $A$, and the choice function $C$ assigns to each finite set of options $o \in O$ a subset of $o, C(o)$. Arrow (1959) stated the following principle of choice (C), and the constraints (A1) and (A2) to assure that the choice function behaves in a 'consistent' way':

(C) $\forall o \in O: C(o) \neq \emptyset$.

(A1) If $o \subseteq o^{\prime}$, then $o \cap C\left(o^{\prime}\right) \subseteq C(o)$.

(A2) If $o \subseteq o^{\prime}$ and $o \cap C\left(o^{\prime}\right) \neq \emptyset$, then $C(o) \subseteq C\left(o^{\prime}\right)$.

If we say that $x>y$, iff $\operatorname{def}_{x} x \in C(\{x, y\})$ and $y \notin C(\{x, y\})$, one can easily show that the ordering as defined above gives rise to a weak order. A structure $\langle I, R\rangle$, with $R$ a binary relation on $I$, is a weak order just in case $R$ is irreflexive (IR), transitive (TR), and almost connected (AC). ${ }^{2}$

Definition 1 A weak order is a structure $\langle I, R\rangle$, with $R$ a binary relation on $I$ that satisfies the following conditions:

(IR) $\forall x: \neg R(x, x)$.

(TR) $\forall x, y, z:(R(x, y) \wedge R(y, z)) \rightarrow R(x, z)$.

(AC) $\forall x, y, z: R(x, y) \rightarrow(R(x, z) \vee R(z, y))$.

If we now define the indifference relation, ' $\sim$ ', as follows: $x \sim y$ iff $_{\text {def }}$ neither $x>y$ nor $y>x$, it is clear that ' $\sim$ ' is an equivalence relation. It is well-known that in case ' $>$ ' gives rise to a weak order, it can be represented numerically by a real valued function $u$ such that for all $x, y \in I: x>y$ iff $u(x)>u(y)$, and $x \sim y$ iff $u(x)=u(y)$.

Within the standard model of economics, an agent is taken to be a 'rational man' if his behavior can be abstractly described in terms of a choice function that satisfies conditions (C), (A1), and (A2).

This standard model has been criticized in economics and gave rise to approaches of bounded rationality. Two standard assumptions of rationality will be given up in this paper. First, the idea that agents are utility optimizers. Second, the idea that the relation of 'indifference' gives rise to an equivalence relation. As for the first, the notion of 'rational man' came under attack in the writings of Herbert Simon in

\footnotetext{
${ }_{1}$ Arrow (1959) actually stated (C) and (A): If $o \subseteq o^{\prime}$ and $o \cap C\left(o^{\prime}\right) \neq \emptyset$, then $C(o)=o^{\prime} \cap C\left(o^{\prime}\right)$. But, obviously, (A) is the combination of (A1) and (A2).

2 In the economic literature, the property of being almost connected is normally called negative transitivity and stated as follows: $\forall x, y, z:(\neg R(x, z) \wedge \neg R(z, y)) \rightarrow \neg R(x, y)$. Obviously, this is just equivalent with (AC).
} 
the 1950s. He claimed that individuals do not necessarily look for the best alternative(s) in a feasible set, but rather that they accept alternatives which they consider satisfactory, i.e., they have a satisficing rather than an optimizing behavior. Tyson (2003) argues that to account for Simon's satisficing behavior, we have to give up on axiom (A1). His argument is that (A1) implements the cognitive assumption that the decision maker fully perceives his preferences among available alternatives. Another way of thinking of Simon's criticism is that we should not seek to derive the meaning of 'better than' in terms of the meaning of 'best' - as is assumed if agents are taken to be utility maximizers - but rather to derive the meaning of 'better than' in terms of the context-dependent meaning of 'good' ${ }^{3}$ What is crucial for the interpretation of the results of our paper is that especially axiom (A1) seems much too strong on such a view: (A1) demands that if both $x$ and $y$ are considered to be good in the context of $\{x, y, z\}$, both should considered to be good in the context $\{x, y\}$ as well. But on a context dependent meaning of 'good', ${ }^{4}$ we want it to be possible that only $x$, or only $y$, is considered to be good in context $\{x, y\}$. We should conclude that if we want to characterize the behavior of 'good', we should give up on (A1). Unfortunately, by just constraints (C) and (A2) we cannot guarantee that the comparison relation 'better than' is as desired. In particular, we cannot guarantee that it is almost connected.

To assure that the comparison relation is as desired, we can add to (C) and (A2) the Upward Difference-constraint (UD), proposed by van Benthem (1982). To state this constraint, we define the notion of a difference pair: $\langle x, y\rangle \in D(o)$ iff $_{\text {def }} x \in C(o)$ and $y \in(o-C(o))$. Now we can define the constraint:

$$
o \subseteq o^{\prime} \text { and } D\left(o^{\prime}\right)=\emptyset \text {, then } D(o)=\emptyset
$$

In fact, van Benthem (1982) states the following constraints: No Reversal (NR), Upward Difference (UD), and Downward Difference (DD) (where $o^{2}$ abbreviates $o \times o$, and $\left.D^{-1}(o)={ }_{\text {def }}\{\langle y, x\rangle:\langle x, y\rangle \in D(o)\}\right)$ :

(NR) $\forall o, o^{\prime} \in O: D(o) \cap D^{-1}\left(o^{\prime}\right)=\emptyset$.

(UD) $o \subseteq o^{\prime}$ and $D\left(o^{\prime}\right)=\emptyset$, then $D(o)=\emptyset$.

(DD) $o \subseteq o^{\prime}$ and $D(o)=\emptyset$, then $D\left(o^{\prime}\right) \cap o^{2}=\emptyset$.

van Benthem (1982) shows that if constraints (NR), (UD) and (UD) are satisfied, the relations ' $\sim$ ' and ' $>$ ' as defined before still have the same properties as before: ' $~$ ' is still predicted to be an equivalence relation, while the relation ' $>$ ' is still predicted to

\footnotetext{
3 Interestingly enough, this is exactly analogue to what Klein (1980) intended to do in linguistics: the meaning of 'taller than' (or 'better than') should be defined in terms of the meaning of 'tall' (or 'good'), not that of 'tallest' (or 'best').

4 You might interpret 'satisficing behavior' in another way: $x$ is good, if it meets some fixed, context independent criteria of acceptability. I find this context independent notion of 'good' rather uninteresting, however.
} 
be (i) irreflexive, (ii) transitive, and (iii) almost connected. It is almost immediate that in case $C$ picks an element of each $o \in O$, instead of a subset, the resulting ordering will also be connected (satisfy for each $x, y \in I: x>y, y>x$, or $x=y$ ) and thus be a linear order.

\section{Semi orders and interval orders}

The 'indifference'-relation induced by the preference order derived in the previous section is transitive, just like the indifference relation induced by preference orders on which the standard theory of choice is based. Already in the 1930s, economists like Armstrong claimed that a basic assumption of the classic utility model, namely, the transitivity of the indifference relation, is highly arguable. A well-known version of this example is due to Luce (1956):

A person may be indifferent between 100 and 101 grains of sugar in his coffee, indifferent between 101 and 102,..., and indifferent between 4999 and 5000 .

If indifference were transitive he would be indifferent between 100 and 5000 grains, and this is probably false.

It is clear that the non-transitivity of indifference results from the inability of human beings to discriminate close quantities. In fact, problems of such kind were already discussed by philosophers in ancient Greece in the so-called 'paradox of the heap', the famous problem induced by vague expressions.

Luce (1956) accounted for the intransitivity of indifference or indiscrimination relations according to which the individual has an (ordinal) real-valued function $u$ defined on the set of alternatives and there exists a given non-negative quantity $\epsilon$ called the treshold. When the individual has to choose from a subset $o$ of feasible alternatives, he chooses the alternative $y$ such there does not exist an $x$ with $u(x)>u(y)+\epsilon$. This model is called a treshold utility model. This treshold utility model corresponds with a model based on a preference relation. This preference relation is not a weak order, but rather what Luce (1956) calls a semi-order. A structure $\langle I, R\rangle$, with $R$ a binary relation on $I$, is a semi-order just in case $R$ is irreflexive (IR), semitransitive (STr), and satisfies the interval-order (IO) condition. A structure that satisfies (IR) and (IO) is called an Interval order. A (strict) partial order is, of course, an order that is irreflexive and transitive.

Definition 2 An Interval order is a structure $\langle I, R\rangle$, with $R$ a binary relation on $I$ that satisfies the following conditions:

(IR) $\forall x: \neg R(x, x)$.

(IO) $\forall x, y, v, w:(R(x, y) \wedge R(v, w)) \rightarrow(R(x, w) \vee R(v, y))$.

Definition 3 A semi order is a structure $\langle I, R\rangle$, with $R$ a binary relation on $I$ that satisfies the following conditions: 
(IR) $\forall x: \neg R(x, x)$.

(IO) $\forall x, y, v, w:(R(x, y) \wedge R(v, w)) \rightarrow(R(x, w) \vee R(v, y))$.

$(\mathrm{STr}) \forall x, y, z, v:(R(x, y) \wedge R(y, z)) \rightarrow(R(x, v) \vee R(v, z))$.

It is easy to see that if $\langle I, R\rangle$ is an interval order, $\langle I, R\rangle$ is a (strict) partial order as well. But this means that weak orders are stronger than semi-orders, which are stronger than interval orders, which in turn are stronger than strict partial orders. Semi-orders are obviously relevant for economics, psychology, but also for linguistics and philosophy as they deal with the analysis of vagueness. The same is true for Interval orders, which are argued also to be relevant for the representation of events (e.g. Wiener 1914; Thomason 1984). Just like Arrow (1959) and van Benthem (1982) did for weak orders, it would be nice if we could characterize semi-orders and interval orders in terms of the behavior of optimizing (Arrow) or satisficing (van Benthem) choice functions among sets of options. Jamison and Lau (1973), Fishburn (1975), and Schwarz (1976) and in a rather different but perhaps more intuitive way, Bandyopadhyay and Sengupta (1993) already showed how to solve the former problem. ${ }^{5}$ It is still an open issue, however, how to derive semi-orders (and interval orders) in terms of satisficing rather than optimizing behavior. The main aim of this paper is to solve this open issue. In fact, two different characterizations will be discussed: what I will call a $K$-characterization and a $P$-characterization. ${ }^{6,7}$

\section{The $K$-characterization}

Our derivation/characterization of several preference orders makes use of two choice functions, that intuitively pick the good and the bad elements. Let us say that $C(o)$ selects the elements of $o$ that are (clearly) good, while $\bar{C}(o)$ selects the elements that are (clearly) bad. Let us define the pairs of elements of $o$ of which the first element is good and the second element bad by $D_{C \bar{C}}(o)={ }_{\operatorname{def}}\{\langle x, y\rangle: x \in C(o) \wedge y \in \bar{C}(o)\}$, and similarly for $D_{\bar{C} C}(o)$. We also define $D_{C N}(o)$ to be the set of ordered pairs of which the first elements is good and the second element is neither good nor bad: $D_{C N}(o)={ }_{\text {def }}\{\langle x, y\rangle: x \in C(o) \wedge y \notin C(o) \wedge y \notin \bar{C}(o)\}$, and similarly for $D_{N C}(o)$, $D_{N \bar{C}}(o)$, and $D_{\bar{C} N}(o)$. In terms of these notions, we can define the set of upward and downward difference pairs:

\footnotetext{
${ }^{5}$ For a paper that addresses a slightly different, but still related question, see Kim (1987).

6 The ' $K$ ' stands for 'Klein, because this characterization is motivated by Klein's (1980) analysis of comparatives in natural language. The ' $P$ ' stands for 'Pragmatic', because it is motivated by the pragmatic constraint that only those subsets $o$ of $A$ are appropriate if $o$ is not closed under $\sim$.

${ }^{7}$ I suggested in this section that there are alternative ways of deriving weak orders in terms of satisficing choice functions. This suggest that there are more alternative ways of deriving semi-orders in terms of satisficing choice functions than I give in the following sections as well. I believe, however, that these alternative ways will be very similar to the ones that I give in this paper.
} 


$$
\begin{aligned}
& D^{\uparrow}(o)=\operatorname{def}_{C \bar{C}} D_{C}(o) \cup D_{C N}(o) \cup D_{N \bar{C}}(o) . \\
& D^{\downarrow}(o)=\operatorname{def}_{\bar{C} C}(o) \cup D_{N C}(o) \cup D_{\bar{C} N}(o) .
\end{aligned}
$$

Now we can give the following four constraints:

$\left(\mathrm{C}^{*}\right) \forall o \in O: C(o) \cap \bar{C}(o)=\emptyset$.

$\left(\mathrm{NR}^{*}\right) \forall o, o^{\prime}: D^{\uparrow}(o) \cap D^{\downarrow}\left(o^{\prime}\right)=\emptyset$.

$\left(\mathrm{UD}^{*}\right)$ If $o \subseteq o^{\prime}$ and $D_{C \bar{C}}\left(o^{\prime}\right)=\emptyset$, then $D_{C \bar{C}}(o)=\emptyset$.

$\left(\mathrm{DD}^{*}\right)$ If $o \subseteq o^{\prime}$ and $D_{C \bar{C}}(o)=\emptyset$, then $D_{C \bar{C}}\left(o^{\prime}\right) \cap o^{2}=\emptyset$.

Constraints (UD*) and (DD*) are very similar to the earlier Upward and Downward Difference constraints of van Benthem (1982), while (C*) assures that $C$ and $\bar{C}$ behave as contraries. The crucial difference with van Benthem's characterization of weak orders is due to the No Reversal constraint $\left(\mathrm{NR}^{*}\right)$, which is much weaker now, due to our use of two, instead of one choice function. We define the preference relation as follows: $x>y$ iff $_{\text {def }} x \in C(\{x, y\})$ and $y \in \bar{C}(\{x, y\})$. Then we can prove that the preference relation is irreflexive and transitive, but it need not satisfy almost connectedness: If $x>y$, it is possible that neither $x>z$ nor $z>y$, because (DD*) doesn't require either of them to hold if $C(\{x, y, z\})=\{x\}$ and $\bar{C}(\{x, y, z\})=\{y\}$.

Now we can prove the following theorem:

Theorem 1 Any choice structure $\langle A, O, C, \bar{C}\rangle$ with $A$ and $O$ as defined above such that $C$ and $\bar{C}$ obey axioms $\left(C^{*}\right),\left(N R^{*}\right),\left(U D^{*}\right)$, and $\left(D D^{*}\right)$, gives rise to a semi order $\langle A,>\rangle$, if we define $x>y$ as $x \in C(\{x, y\})$ and $y \in \bar{C}(\{x, y\})$.

In order to show that the above constraints guarantee that the induced ordering is a semi-order, we have to show that the resulting comparison relation is irreflexive and that it satisfies (IO) and (STr). Irreflexivity follows immediately from the definition of the comparison relation together with constraint $\left(\mathrm{C}^{*}\right){ }^{8}$

Proof of $(I O)$ Assume $x>y$ and $v>w$. Because $x>y$, the following cases are possible for $\langle x, y, w\rangle$ (with (NR*) and (UD*)): $C \overline{C C}, C C \bar{C}, C N \bar{C}, C \bar{C} C, N \bar{C} C, \overline{C C} C$, and $C \bar{C} N$. From the first three options, we immediately conclude with (DD*) that $x>w$. From the fourth, fifth, and sixth options, we conclude that $w>y$, from which we can easily derive with $v>w$ and transitivity that $v>y$. Thus, the only possibility that does not satisfy the consequent of (IO) is $C \bar{C} N$. By parallel reasoning, the only possibility for $\langle v, w, y\rangle$ that doesn't satisfy the consequent of (IO) is $C \bar{C} N$

\footnotetext{
8 Transitivity can be proved as follows: Suppose $x>y$ and $y>z$, now look at $\langle x, y, z\rangle$. Then we have the following possibilities not ruled out by (UD*): $C \bar{C} C, C \bar{C} N, \overline{C C} C, \bar{C} N C, N \bar{C} C$, and $N C \bar{C}$, which are all in contradiction with (NR*), and $C C \bar{C}, C \overline{C C}$, and $C N \bar{C}$, from which we can derive via (DD*) that $x>z$. It follows that $x>z$, which means that $>$ is transitive. But of course, transitivity follows if the comparison relation is irreflexive and satisfies (IO).
} 
(because $v>w$ ). Thus, the only possibility that doesn't satisfy (IO) at all is where both $\langle x, y, w\rangle$ and $\langle v, w, y\rangle$ behave as $C \bar{C} N$. But now $\langle y, w\rangle \in D^{\uparrow}(\{v, w, y\})$ and $\langle y, w\rangle \in D^{\downarrow}(\{x, y, w\})$, which is ruled out by $\left(\mathrm{NR}^{*}\right)$.

The above reasoning shows that the induced ordering gives rise to an interval-order. On top of that, the ordering will also be a semi-order if it also satisfies semi-transitivity, (STr). We prove this as follows: assume $x>y$ and $y>z$. Because $x>y$, the following cases are possible for $\langle x, y, v\rangle$ (with (NR*) and (UD*)): $C \overline{C C}, C C \bar{C}, C N \bar{C}, C \bar{C} C$, $N \bar{C} C, \overline{C C} C$, and $C \bar{C} N$. From the first three options we immediately conclude with (DD*) that $x>v$. From the fourth, fifth, and sixth options, we conclude that $v>y$, from which we can easily derive with $y>z$ and transitivity that $v>z$. Thus, the only possibility that doesn't satisfy the consequent of (STr) is $C \bar{C} N$. By parallel reasoning, the only possibility for $\langle y, z, v\rangle$ is $C \bar{C} N$ (because $y>z$ ). But now $\langle y, v\rangle \in D^{\downarrow}(\{x, y, v\})$ and $\langle y, v\rangle \in D^{\uparrow}(\{y, z, v\})$, which is ruled out by $\left(\mathrm{NR}^{*}\right)$. Conclusion: the comparison relation generated by our four constraints gives rise to a semi-order.

It is also possible to show something stronger than Theorem 1 , i.e., that our constraints in fact characterize semi-orders. To do that, we have to show that from each semi-order we can define a choice structure $\langle A, O, C, \bar{C}\rangle$ that satisfies the above constraints. Let $\langle I, R\rangle$ be a semi-order. We define $\langle A, O, C, \bar{C}\rangle$ as follows: $A=I$ and $O$ is the totality of its finite subsets. For the definitions of $C$ and $\bar{C}$, we look for each $o \in O$ at the maximal and minimal objects in $o$ with respect to the ' $\geq$ '-relation. Choice function $C$ picks the maximal object(s) in $o,\{x \in c: \forall y \in c: x \geq y\}$, while $\bar{C}$ picks the minimal objects in $o$. In case each pair of objects in $o$ are all mutually $\sim$-related, $C(o)=o($ or $\bar{C}(o)=o$.) It is easy to see that the generated structure satisfies the constraints.

In terms of two instead of one choice functions, we can also derive/characterize other ordering relations. In order to do so, we define the following new constraints $\left(\mathrm{C}^{* *}\right),\left(\mathrm{NR}^{* *}\right)$, and $\left(\mathrm{UD}^{* *}\right)$ :

$$
\begin{aligned}
& \left(\mathrm{C}^{* *}\right) \forall o \in O: C(o) \cap \bar{C}(o)=\emptyset \text { and } C(o) \cup \bar{C}(o)=o . \\
& \left(\mathrm{NR}^{* *}\right) \forall o, o^{\prime} \in O: a, b \in\{C, \bar{C}, N\}: a \neq b \rightarrow D_{a b}(o) \cap D_{b a}\left(o^{\prime}\right)=\emptyset . \\
& \left(\mathrm{UD}^{* *}\right) \text { If } o \subseteq o^{\prime} \text { and }\langle x, y\rangle \in D_{C \bar{C}}(o) \text {, then } D_{C \bar{C}}\left(o^{\prime}\right) \neq \emptyset \text { and } \\
& \langle x, y\rangle \notin D^{\downarrow}\left(o^{\prime}\right) .
\end{aligned}
$$

Notice that $\left(\mathrm{C}^{* *}\right)$ and $\left(\mathrm{UD}^{* *}\right)$ strengthen $\left(\mathrm{C}^{*}\right)$ and $(\mathrm{UD}) /\left(\mathrm{UD}^{*}\right)$ respectively, while $\left(\mathrm{NR}^{* *}\right)$ is stronger than $(\mathrm{NR})$, but weaker than $\left(\mathrm{NR}^{*}\right)$. Notice also that in the context of $\left(\mathrm{NR}^{*}\right),\left(\mathrm{UD}^{* *}\right)$ reduces to (UD)/(UD*). Now we can state the following theorems:

Theorem 2 Any choice structure $\langle A, O, C, \bar{C}\rangle$ with $A$ and $O$ as defined above such that $C$ and $\bar{C}$ obey axioms $\left(C^{*}\right),(N R),(D D)$ and $\left(U D^{* *}\right)$, gives rise to a (strict) partial $\operatorname{order}\langle A,>\rangle$, if we define $x>y$ as $x \in C(\{x, y\})$ and $y \in \bar{C}(\{x, y\})$. 
Theorem 3 Any choice structure $\langle A, O, C, \bar{C}\rangle$ with $A$ and $O$ as defined above such that $C$ and $\bar{C}$ obey axioms $\left(C^{*}\right),\left(U D^{*}\right),\left(D D^{*}\right)$ and $\left(N R^{* *}\right)$, gives rise to an interval $\operatorname{order}\langle A,>\rangle$, if we define $x>y$ as $x \in C(\{x, y\})$ and $y \in \bar{C}(\{x, y\})$.

Theorem 4 Any choice structure $\langle A, O, C, \bar{C}\rangle$ with $A$ and $O$ as defined above such that $C$ and $\bar{C}$ obey axioms $\left(C^{* *}\right),\left(N R^{*}\right),\left(U D^{*}\right)$, and $\left(D D^{*}\right)$, and where gives rise to a weak order $\langle A,>\rangle$, if we define $x>y$ as $x \in C(\{x, y\})$ and $y \in \bar{C}(\{x, y\}){ }^{9}$

The proofs of these theorems are relatively simple. To prove Theorem 4 , for instance, it is enough to observe that in case $\left(\mathrm{C}^{* *}\right)$ holds, the constraints $\left(\mathrm{NR}^{*}\right),\left(\mathrm{UD}^{*}\right)$, and (DD*) reduce to (NR), (UD), and (DD), and we are back to van Benthem's characterization of weak orders.

In order to prove Theorem 2, we have to show that $>$ is irreflexive and transitive. The former is true by definition, and to prove the latter, assume $x>y$ and $y>z$. Given the constraints $\left(\mathrm{C}^{*}\right),(\mathrm{NR}),(\mathrm{DD})$ and $\left(\mathrm{UD}^{* *}\right)$, this means that the following cases are possible for $\langle x, y, z\rangle: C \overline{C C}, C C \bar{C}, C N \bar{C} .{ }^{10}$ From all three and (DD*) we conclude that $x>z$. Notice that the constraints do not guarantee that the interval order condition (IO), semi transitivity (STr), and almost connectedness (AC) are obeyed. (IO): if $x>y$ and $v>w$ it is possible that $\langle x, y, w\rangle$ be $C \bar{C} N$ and that $\langle v, w, y\rangle$ be $C \bar{C} N$ without deriving a contradiction. (STr): if $x>y$ and $y>z$, it is possible for cases $\langle x, y, v\rangle$ and $\langle y z v\rangle$ to be $C \bar{C} N$ without deriving a contradiction. (AC): if $x>y$ it is possible for case $\langle x, y, v\rangle$ to be $C \bar{C} N$ without deriving a contradiction.

To prove Theorem 3, we have to prove that (IO) holds. So suppose $x>y$ and $v>w$. Because $x>y$, the following cases are possible for $\langle x, y, w\rangle$ (with $\left(\mathrm{NR}^{* *}\right.$ ) and (UD*)): $C \overline{C C}, C \bar{C} \bar{C}, C N \bar{C}, C \bar{C} C, N \bar{C} C, \overline{C C} C$, and $C \bar{C} N$. From the first three options, we immediately conclude with (DD*) that $x>w$. From the fourth, fifth, and sixth options, we conclude that $w>y$, from which we can easily derive with $v>w$ and transitivity that $v>y$. Thus, the only possibility that doesn't satisfy the consequent is $C \bar{C} N$. By parallel reasoning, the only possibility for $\langle v, w, y\rangle$ that doesn't satisfy the consequent of (IO) is $C \bar{C} N$ (because $v>w$ ). Thus, the only possibility that doesn't satisfy (IO) at all is where both $\langle x, y, w\rangle$ and $\langle v, w, y\rangle$ behave as $C \bar{C} N$. But now $\langle y, w\rangle \in D_{\bar{C} N}(\{v, w, y\})$ and $\langle y, w\rangle \in D_{N \bar{C}}(\{x, y, w\})$, which is ruled out by $\left(\mathrm{NR}^{* *}\right)$. Notice that the constraints do not guarantee that semi transitivity (STr) and almost connectedness (AC) are obeyed. (STr): if $x>y$ and $y>z$, it is possible for cases $\langle x, y, v\rangle$ and $\langle y, z, v\rangle$ to be $C \bar{C} N$ without deriving a contradiction. (AC): if $x>y$ it is possible for $\langle x, y, v\rangle$ to be $C \bar{C} N$ without deriving a contradiction.

\section{The $P$-characterization}

In the previous section we assumed that all finite subsets of $A$ are proper sets of options. In this section we will derive semi-orders based on a somewhat different intuition. The

\footnotetext{
9 This theorem combined with Theorem 1 is used in van Rooij (2010) to account for the distinction between explicit ('John is taller than Mary') versus implicit ('John is tall compared to Mary') comparatives.

10 Notice that cases $C \bar{C} N, N C \bar{C}$ and $\bar{C} N C$ are ruled out by (UD**).
} 
idea is that if an agent is indifferent between $x$ and $y$ (perhaps because they are indistinguishable), the set $\{x, y\}$ is not a suitable set of options, and thus not an element of $O$. According to our new characterization of semi-orders, we just take the constraints of van Benthem (1982), but don't require that $O$ consists of all finite subsets of $A$. A happy consequence of giving up this constraint is that almost-connectedness is no longer valid, which is the main difference between weak and semi-orders. Unfortunately, however, just dropping the constraint means that transitivity of the comparison relation is given up as well. van Benthem (1982) already suggested that transitivity can be restored by putting a closure conditions on $O$. In the spirit of this suggestion, we propose to characterize semi-orders by adding some other closure conditions. The idea is that we just start with all subsets of $O$ that consist of two elements and for which (U) holds, and close this set of subsets of $O$ under closure condition (P1):

(U) $\forall o \in O$ : if $\operatorname{card}(o)=2$, then $\emptyset \neq C(o) \neq o$.

(P1) $\forall o \in O: \forall a \in \bigcup O: o \cup\{a\} \in G A P \rightarrow o \cup\{a\} \in O$.

One might think of condition (U) as a usefulness condition on $C$ for elements of $O$ which contain only two options. Constraint (P1) says that to any element $O$ of $O$ one can add any element $a \in A$ to it that is in an ordering relation with respect to at least one other element, on the condition that the resulting set $o \cup\{a\}$ satisfies constraint $G A P$. To state this constraint, suppose that $o$ contains $n(>2)$ elements (written by $o^{n}$ ). Then the constraint says that there must be at least $n-1$ subsets $o^{\prime}$ of $o$ with cardinality $n-1$ such that all these $o^{\prime}$ are also elements of $O$.

$$
o^{n} \in G A P \quad \text { iff }_{d e f} \quad \exists_{n-1} o^{\prime} \subset o: \operatorname{card}\left(o^{\prime}\right)=n-1 \wedge o^{\prime} \in O .
$$

The intuition behind this condition is that only those subsets of $A$ satisfy GAP if there is at least one gap in this subset w.r.t. the relevant property. It is easy to show that this closure condition guarantees that the resulting ordering relation will satisfy transitivity and will thus be a strict partial order. Suppose that $x>y$ and $y>z$. By construction this means that $\{x, y\}$ and $\{y, z\}$ are both in $O$. Now take $\{x, y\}$ and ask whether we can add $z$ to it such that the result, $\{x, y, z\}$, is still in $O$. This is the case if $\{x, y, z\} \in G A P$, and thus if $\{x, y, z\}$ has two subsets of cardinality 2 which are in $O$. But we know already that this is the case: $\{x, y\}$ and $\{x, z\}$. Given that $x>y$, it follows that the only possibilities for $\{x, y, z\}$ that are allowed (by using (DD)) are $T T S, T S S, T S T$, and $S S T$. Given that $y>z$, the only ones that are left (after using (DD)) are $T T S$ and $T S S$. By (DD) we can now conclude that $x>z$. So, we have proved the following theorem:

Theorem 5 Any choice structure $\langle A, O, C\rangle$ with A a set of options, where $C$ obeys axioms (NR), (DD), (UD), and $(U)$, and where $O$ is closed under $(P 1)$, gives rise to a strict partial order $\langle A,>\rangle$, if we define $x>y$ as $x \in C(\{x, y\})$ and $y \notin C(\{x, y\})$. 
Constraint (P1) doesn't guarantee yet that the ordering relation satisfies the Interval order condition (IO) $(\forall x, y, v, w:(x>y \wedge v>w) \rightarrow(x>w \vee v>y))$ or semi-transitivity. To see the first, suppose that $x>y$ and $v>w$. This means that $O$ contains at least the following sets: $\{x, y\}$ and $\{v, w\}$. If the four elements are all different, however, $(\mathrm{P})$ doesn't demand that any other set belongs to $O$. In particular, it doesn't demand that $\{x, y, v\}$ or $\{x, y, w\}$ belongs to $O$. But this is exactly what is required for the resulting ordering relation to satisfy the Interval order condition. So, we add the following condition:

(OR) $\forall o \in O,\{x, y\} \in O: o \cup\{x\} \in O$ or $o \cup\{y\} \in O$.

It is easy to prove that this suffices for the resulting ordering relation to satisfy (IO). Suppose $x>y$ and $v>w$. This means that $O$ contains at least the following sets: $\{x, y\}$ and $\{v, w\}$. By (OR) this means that either $\{v, w, x\} \in O$ or $\{v, w, y\} \in O$. Suppose $\{v, w, x\} \in O$. It follows by $v>w$, (NR), and (DD) that only the following possibilities are allowed for this set: $T T S, T S S, T S T$, and $S S T$. By (DD) this means that either $v>x$ or $x>w$. But we know already that the ordering relation is transitive (if (P1) is assumed), it follows by $x>y$ that $v>y$ or $x>w$, which was what we needed to prove. A similar argument shows that if $\{v, w, y\} \in O$ it follows that $v>y$ or $x>w$, and so we proved what we wanted: the resulting ordering relation satisfies the Interval ordering condition.

Theorem 6 Any choice structure $\langle A, O, C\rangle$ with A a set of options, where $C$ obeys axioms (NR), (DD), (UD), and $(U)$, and where $O$ is closed under $(P 1)$, and $(O R)$, gives rise to an interval order $\langle A,>\rangle$, if we define $x>y$ as $x \in C(\{x, y\})$ and $y \notin C(\{x, y\})$.

The resulting ordering relation still does not necessarily satisfy semi-transitivity: $\forall x, y, z, v:(x>y \wedge y>z) \rightarrow(x>v \vee v>z)$. For suppose $x>y$ and $y>z$. By (P1) it follows that $\{x, y, z\} \in O$, and thus that $\{x, z\} \in O$ and $x>z$. But nothing requires for any arbitrary $v$ to be related in any way to any other element. If $A=\{x, y, z, v\}, O$ might well just be the following set: $\{\{x, y\},\{y, z\},\{x, z\},\{x, y, z\}\}$, and then it will be the case that neither $x>v$ nor $v>z$ holds. To assure that the resulting ordering also satisfies semi-transitivity, we propose the following closure condition on $O$ :

(P2) $\forall o \in O, a \in A: o \in G A P_{2} \rightarrow o \cup\{a\} \in O$, where

$o^{n} \in G A P_{2} \quad$ iff $_{d e f} \quad \exists_{n} o^{\prime} \subset o: \operatorname{card}\left(o^{\prime}\right)=n-1 \wedge o^{\prime} \in O$.

Notice the subtle difference between $G A P_{2}$ and $G A P$ : whereas the former requires that there are at least $n$ subsets of $o^{n}$ in $O$ with cardinality $n-1$, the latter requires this only for $n-1$ subsets. The intuition between this formal difference is that whereas $o$ satisfies $G A P$ already if it contains at least one gap, $o$ can only satisfy $G A P_{2}$ if it has at least $t w o$ gaps. Consider subsets of the natural numbers and assume that such a subset has a gap if at least one number in the order is missing. Thus, $\{1,2,3,4\}$ has 
no gap, $\{1,2,3,5\}$ has 1 , but $\{1,3,4,6\}$ and $\{1,3,5,7\}$ have two or more gaps. The set $\{1,2,3,5\}$ has the following subsets of 3 numbers with a gap: $\{1,2,5\},\{1,3,5\}$, and $\{2,3,5\}$. Thus it has 3 such subsets, which means that $\{1,2,3,5\}$ satisfies $G A P$, but not $G A P_{2}$. The set $\{1,3,4,6\}$, on the other hand, has 4 four subsets of 3 elements with a gap: $\{1,3,4\},\{1,3,6\},\{1,4,6\}$, and $\{3,4,6\}$ which means that it satisfies both $G A P$ and $G A P_{2}$. The same holds for the set $\{1,3,5,7\}$. The idea behind constraint (P2) is that to the set $\{1,3,4,6\}$ we can always add an arbitrary natural number and still have a gap (and thus be an appropriate set of options), but that this doesn't hold for $\{1,2,3,5\}$ : adding 4 would result in an inappropriate set of options. As far as I can see, the closure conditions defined above are such that they generate all subsets of $A$ for which there is such a sufficiently large gap.

Now we prove that if $O$ is closed under (P2), the resulting ordering relation satisfies semi-transitivity. Suppose $x>y$ and $y>z$. By (P1) it follows that $\{x, y, z\} \in$ $O$ and by (DD) that also $\{x, z\} \in O$. But this means that $\{x, y, z\}$ has 3 subsets of two elements which are in $O$, and thus that $\{x, y, z\}$ satisfies $G A P_{2}$. By (P2) this means that $\{x, y, z, v\}$ is also in O. Given that the only constellations possible for $\{x, y, z\}$ are $T T S$ and $T S S$, the only possible constellations for $\{x, y, z, v\}$ are $T T T S, T T S S, T S S S, T T S T, T S S T$, and SSST. If one of the first three holds it follows from (DD) that $x>v$. If one of the last three holds, it follows from (DD) that $v>z$. But this means that semi-transitivity is proved.

Theorem 7 Any choice structure $\langle A, O, C\rangle$ with A a set of options, where $C$ obeys axioms (NR), (DD), (UD), and (U), and where $O$ is closed under (P1), (OR), and (P2) gives rise to a semi-order $\langle A,>\rangle$, if we define $x>y$ as $x \in C(\{x, y\})$ and $y \notin C(\{x, y\})$.

It is important to notice that our constraints do not yet guarantee that the resulting ordering is also almost connected, (AC) $\forall x, y, z: x>y \rightarrow(x>z \vee z>y)$. If that were the case, the resulting ordering relation would not only be a semi-order, but also a weak order, which is what we don't want. It suffices to show that there is at least one choice structure satisfying the above constraints which doesn't give rise to a weak order. Assume $A=\{x, y, z\}$ and $x>y$. This means that $\{x, y\} \in O$. But no constraint forces us to assume that any of $\{x, z\},\{y, z\}$, or $\{x, y, z\}$ is in $O$, which is all we need.

\section{Conclusion and outlook}

In this paper I have given a derivation of the meaning of 'better than' from a plausible analysis of the meaning of 'good', instead of the meaning of 'best'. I have argued that the interpretation of these technical results is relevant for the modeling of bounded rational agents. Semi-orders and interval orders are relevant in this respect because they were introduced by Luce (1956) to model the fact that behavior is invariant on just noticeable differences in preference. The axioms that constrain the behavior of the choice functions that models the meaning of 'good' (and 'bad') is argued to be an interpretation of Simon's satisficing, rather than utility maximizing agents. On the 
basis of this interpretation, this paper gives a characterizations of several preference relations in terms of constraints on the behavior of satisficing agents.

In this paper I interpreted the relations ' $>$ ' and ' $\sim$ ' as preference and indifference, respectively. But there is nothing in the formal analysis that requires us to interpret the relations in that way. Two other equally natural interpretations are 'taller than' and 'equally tall as', and 'earlier/later than' and 'simultaneous with'. In fact, the original motivation of this work came from the latter two interpretations (see van Rooij, to appear). I believe that the results of this paper are significant as well for the analysis of natural language comparatives, temporal relations, and more generally, for the analysis of vagueness.

Open Access This article is distributed under the terms of the Creative Commons Attribution Noncommercial License which permits any noncommercial use, distribution, and reproduction in any medium, provided the original author(s) and source are credited.

\section{References}

Arrow, K. (1959). Rational choice functions and orderings. Economica, 26, 121-127.

Bandyopadhyay, T., \& Sengupta, K. (1993). Characterization of generalized weak orders and revealed preference. Economic Theory, 3, 571-576.

Fishburn, P. C. (1975). Semiorders and choice functions. Econometrica, 43, 975-977.

Jamison, D. T., \& Lau, L. J. (1973). Semiorders and the theory of choice. Econometrica, 41, 901-912. Kim, T. (1987). Intransitive indifference and revealed preference. Econometrica, 55, 163-167.

Klein, E. (1980). The semantics of positive and comparative adjectives. Linguistics and Philosophy, 4, 1-45. Luce, R. D. (1956). Semiorders and a theory of utility discrimination. Econometrica, 24, 178-191.

Schwarz, T. (1976). Choice functions, rationality conditions, and variations on the weak axioms of revealed preference. Journal of Economic Theory, 13, 414-427.

Simon, H. (1955). A behavioral model of rational choice. Quarterly Journal of Economics, 59, 98-118. Thomason, S. K. (1984). On constructing instants from events. Journal of Philosophical Logic, 13, 85-96. Tyson, C. (2003). Revealed preference analysis of bounded rational choice, $\mathrm{PhD}$. thesis, Stanford University.

van Benthem, J. (1982). Later than late: On the logical origin of the temporal order. Pacific Philosophical Quarterly, 63, 193-203.

van Rooij, R. (2010). Implicit versus explicit comparatives. In P. Egre \& N. Klinedinst (Eds.), Vagueness and language use. Basingstoke: Palgrave MacMIllan.

van Rooij, R. (to appear). Vagueness and linguistics. To appear In Ronzitti (Ed.), Vagueness: A guide, NY: Springer.

Wiener, N. (1914). A contribution to the theory of relative position. Proceedings of the Cambridge Philosophical Society, 17, 441-449. 